\title{
Notule sur une mystérieuse église byzantine de Kaouvi
}

Le colophon géorgien du manuscrit 'Iviron géorg. 69'

\author{
Sergey Kim \\ Académie théologique de Moscou, Russia; Université de Bâle, Suisse \\ kimserge@gmail.com
}

\begin{abstract}
The note offers a corrected reading of a Georgian colophon in Iviron, georg. 69, that R. P. Blake published in 1932. It is demonstrated that George the Athonite, its author, did not mention a Byzantine church of Kaouvi, as Blake had read. Several literary parallels from other texts connected to George are provided.
\end{abstract}

\section{Keywords}

Georgian manuscripts - Georgian language - colophon - George the Athonite Byzantine churches - hymnography

En conduisant une recherche sur un fragment de Ménée géorgien conservé dans la bibliothèque du monastère Simonopetra au Mont Athos, ${ }^{1}$ je me suis porté à étudier différents colophons qui accompagnent les manuscrits des Ménées liturgiques traduits par Georges l'Hagiorite (†1065). Une grande partie de ces colophons a été éditée et traduite en français par Robert P. Blake dans son catalogue des manuscrits géorgiens du monastère Iviron. ${ }^{2}$ Neuf colophons des

* L'auteur remercie cordialement le prof. Bernard Outtier (CNRS) et Dr. Emanuele Castelli (Université de Bâle) pour une relecture attentive et critique de la présente note.

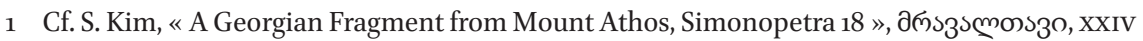
(2015), p. 209-216.

2 R. Blake, Catalogue des manuscrits géorgiens de la bibliothèque de la Laure d'Iviron au Mont Athos, ROC, VIII (28) (1931-1932), pp. 289-361 [1-73] ; IX (29) (1933-1934), pp. 114-159 [74-119] ; IX (29) (1933-1934), pp. 225-271 [120-166]. 
Ménées, certains repris à l'édition de Blake, ont été publiés en appendice à l'ouvrage récent de Lali Djghamaïa. ${ }^{3}$ Certaines de ces annotations sont issues de la main du même saint traducteur, et elles nous fournissent plusieurs informations importantes sur la provenance et la structure des sources byzantines des Ménées géorgiens. ${ }^{4}$

Un de ces colophons est conservé dans le manuscrit Athous, Iviron georg. 69, qui contient le Ménée de novembre (f. 197r) ; rédigé en écriture onciale asomthavrouli, il est un autographe de Georges l'Hagiorite. Il fait partie des matériaux publiés par Robert Blake ${ }^{5}$ et a été analysé par Lali Djghamaïa du point de vue historique. ${ }^{6}$ Je voudrais rouvrir la discussion des informations que Mme Djghamaïa touche dans ses études, suite à l'obtention d'une nouvelle photo du f. 197r de ce manuscrit que nous devons à l'obligeance à la fois du Père Théologos, le bibliothécaire d'Iviron, et des professeurs Jost Gippert et Bernard Outtier, qui ont récemment visité cet illustre monastère athonite.

\section{État de la question}

Dans son catalogue des manuscrits d'Iviron Robert Blake donnait le texte suivant du colophon - pour n'en citer que la partie la plus importante : 3 os

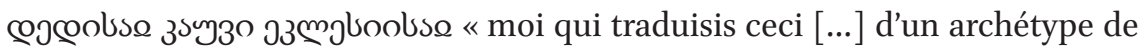
l'église de Kaouvi $(K \alpha \lambda \circ u ́ \beta \iota \alpha) »$. Il accompagnait le nom étrange de Kaouvi d'une rétroversion en grec: $K \alpha \lambda$ ov่ $\beta \iota$, qui ne résolvait pas d'ailleurs le problème d'identification. ${ }^{7}$ Comme bien d'autres colophons insérés dans les Ménées géorgiens, cette annotation avait toutes les apparences d'indiquer la provenance du manuscrit byzantin qui a servi de base pour ce Ménée de novembre. Les lecteurs du catalogue de Blake restaient longtemps intrigués par cette église de зऽэзo, Kaouvi, d'un nom étrange.

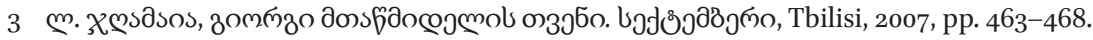

4 Nous espérons bientôt publier une étude sur un cas particulier de correspondance et de divergence entre les Ménées géorgiens et ceux grecs, tiré du Ménée de décembre.

5 Pour une description sommaire cf. : Blake, Catalogue, ROC, IX (29), pp. 255-256 [150-151].

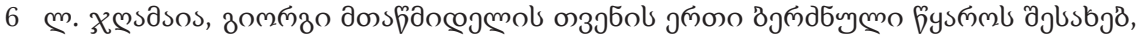

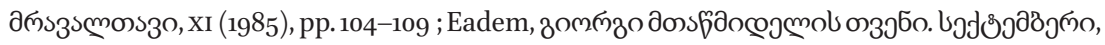
Tbilisi, 2007, pp. 17-19.

7 En effet, le répertoire de Janin donnait des renseignements sur $\tau \dot{\alpha} \mathrm{K} \alpha \lambda \dot{\jmath} \beta 1 \alpha$ à Constantinople (R. Janin, Constantinople byzantine. Développement urbain et répertoire topographique (Archives de l'Orient chrétien, 4), Paris, 1950, p. 339), mais plutôt qu'une église, c'était un quartier. 
En 1985 Lali Djghamaïa ${ }^{8}$ proposa une hypothèse ingénieuse qui liait ce nom

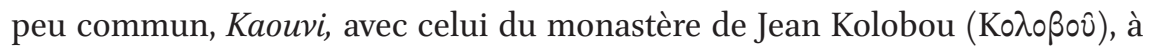
Hiérissos, ville limitrophe de la péninsule d'Athos. En 979/980 cet établissement monastique - anciennement illustre, mais en déclin à la fin du $\mathrm{X}^{\mathrm{e}}$ siècle - devint propriété de la naissante communauté d'Iviron suite aux libéralités de l'empereur Basile II envers les moines géorgiens. ${ }^{9}$ Pour la chercheuse géorgienne l'existence d'un Ménée traduit par Georges l'Hagiorite d'après un exemplaire grec du monastère de Jean Kolobou impliquait deux conclusions : a) le couvent de Kolobou conservait son lien avec les géorgiens à l'époque où saint Georges (†1065) exerçait son activité littéraire; b) le monastère de Kolobou possédait des manuscrits grecs aussi importants que ceux de Sainte-Sophie de Constantinople et de Saint-Syméon près d'Antioche, mentionnés dans d'autres colophons des Ménées de saint Georges. ${ }^{10}$

Comme l'hypothèse de Mme Djghamaïa nous semblait difficile à être acceptée, ${ }^{11}$ nous avions pensé à une autre explication de l'énigme de Kaouvi, avant d'avoir la possibilité de consulter les images du manuscrit. Les caractères ' $\mathrm{g}$ et $7 \mathrm{o}$ étant très proches graphiquement en écriture onciale géorgienne, on

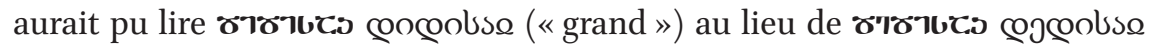
(« original»). Ensuite, on pourrait y soupçonner une abréviation, telle que

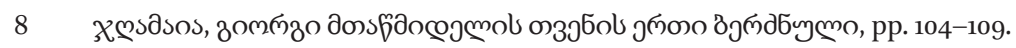

9 Sur les circonstances de ce don on consultera, e.g., les renseignements de la Vie des saints

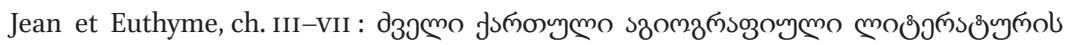

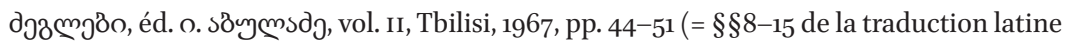
de Paul Peeters, cf. P. Peeters, « Histoires monastiques géorgiennes », AB, 36-37 (19171919), pp. 18-24); on consultera aussi : B. Martin-Hisard, « La Vie de Jean et Euthyme et le statut du monastère des Ibères sur l'Athos », RÉB, 49 (1991), p. 88-93. Cf. aussi P. Peeters, «Un colophon géorgien de Thornik le Moine », $A B$, 50 (1932), pp. 358-371.

10 Les originaux grecs de Sainte-Sophie de Constantinople sont mentionnés dans : Tbilisi, National Center of Manuscripts, S-4999, p. 662, indirectement en Iviron géorg. 65, f. 195v ; ceux de Saint-Syméon près d'Antioche dans : Iviron géorg. 65, f. 195v, Jérusalem, Patriarcat grec, géorg. 98, f. 216r, Tbilisi, National Center of Manuscripts, S-4999, p. 662. Cf. une édition

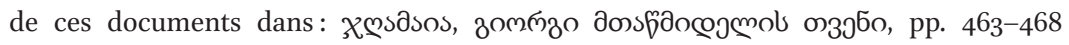
(Appendice III).

11 En effet, l'hypothèse de la chercheuse géorgienne reposait sur la transcription grecque proposée par Robert Blake qui n'était pas en soi satisfaisante. En effet, pour que le grec K $\alpha \lambda$ oú $\beta 1 \alpha$ puisse se transformer en géorgien Kaouvi, il faut que la consonne [l] disparaisse de sa position entre les voyelles [a] et [u], ce qui ne peut être justifié par aucun phénomène phonétique. D’ailleurs, même si l'on acceptait la suggestion de Blake, le vocalisme

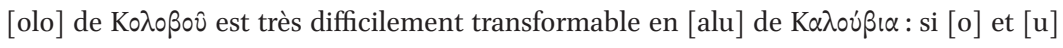
sont interchangeables en géorgien en fonction de l'orthographe des copistes, le passage de $[\mathrm{ol}]$ en [al] est très difficile à admettre soit en grec, soit en géorgien. 
kṕoyli au lieu de kaoyvi. Dans ce cas, il s'agirait de Sainte-Sophie : cocoobse

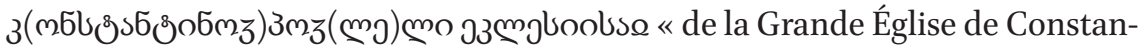
tinople », la ville et l'église mentionnée dans d'autres colophons des Ménée athonites. $^{12}$

Pourtant l'examen des photos du colophon nous a mis en face d'une tout autre réalité.

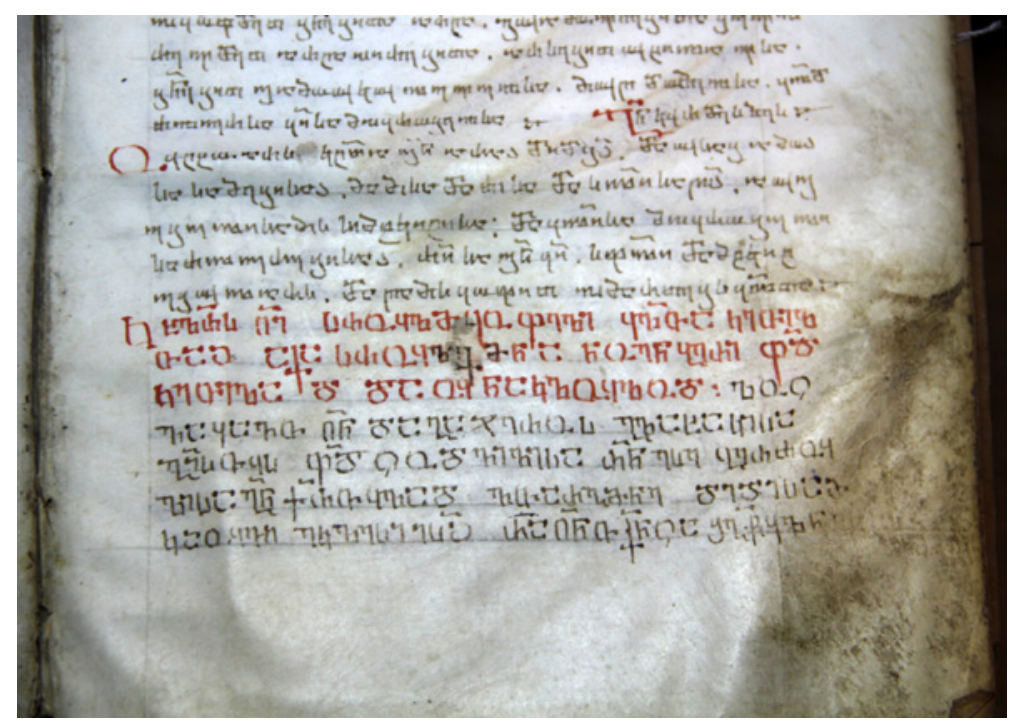

(C) COURTESY OF THE IVIRON MONASTERY, MOUNT ATHOS, GREECE

\section{Nouvelle édition du colophon}

Donnons maintenant la nouvelle édition du texte du colophon de saint Georges. La traduction française est nôtre ; les leçons de Blake se trouvent dans les notes. La mise en paragraphes et la ponctuation sont à nous ; la partie marquée en gras est écrite en rouge dans le codex.

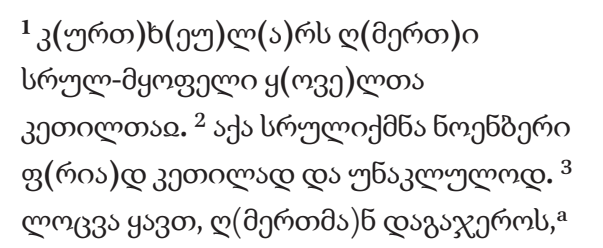

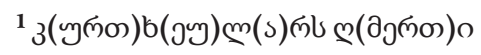

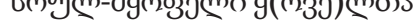
उ

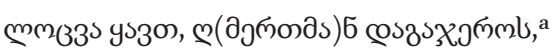

\begin{abstract}
${ }^{1}$ Béni est Dieu qui accomplit tous les biens. ${ }^{2}$ Ici s'achève Novembre, de façon correcte et sans lacunes. ${ }^{3}$ Faites une prière - Dieu vous donnera confiance - pour (moi), pauvre
\end{abstract} Georges, le grand pécheur, 


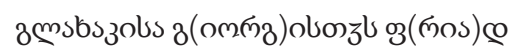

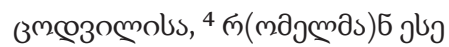

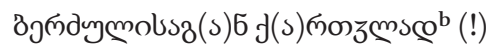

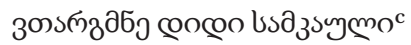

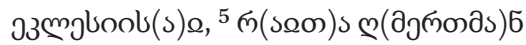

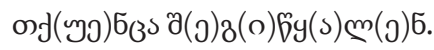

${ }^{4}$ qui traduisis de grec en géorgien ce grand ornement de l'église, ${ }^{5}$ afin que Dieu prenne pitié de vous aussi.

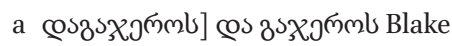

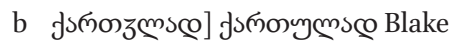

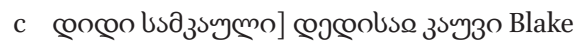

Les corrections infimes que nous proposons aux pages savantes de Blake au-

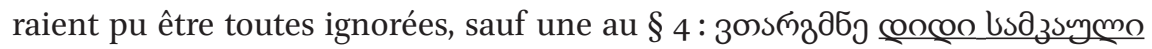

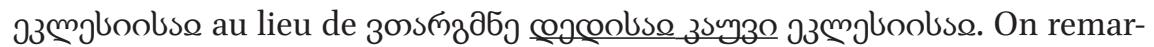

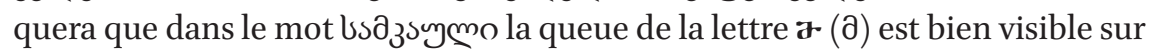
la photo, ce qui exclut un s ( $\Omega$ ), cf. photo, ligne 2 ab imo. Pour ce qui concerne la lettre $\mathbf{b}(\mathrm{m})$ dans le même mot, elle a bien l'apparence d'un $\mathbf{7} \cdot(3)$, comme lue par Blake ; cela est peut-être dû au fait que la lettre 'b a été posée sur la ligne horizontale de la réglure, absorbant ainsi le trait inférieur horizontal du signe. Notons que plus bas, le mot $\partial(\jmath) z(0) \mathscr{b} y(১) m(\jmath) 6$ présente exactement la même situation : la partie inférieure du signe 'b se distingue à peine, si bien que la lettre ressemble fortement à un $\mathbf{7}$ (cf. photo, ligne 1 ab imo). Il s'agit donc du « grand ornement de l'Église » («Église » dans le sens théologique) et non pas de « l'original de l'église de Kaouvi ».

\section{Parallèles littéraires}

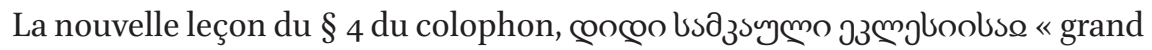
ornement de l'Église » se prête à une analyse littéraire. En effet, elle trouve des parallèles dans un autre colophon de Georges, aussi bien que dans un passage de la Vie de saint Georges dédié à la traduction du Synaxaire. On comparera :

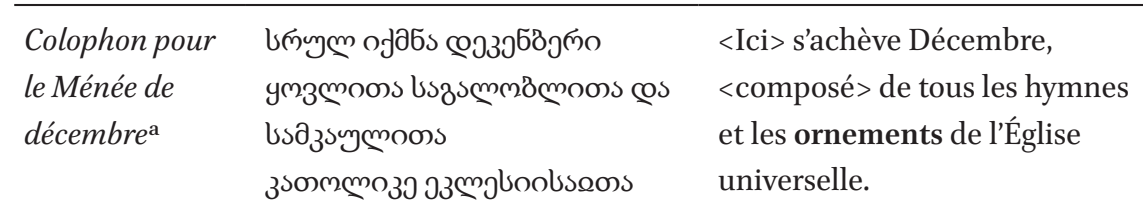




\begin{tabular}{|c|c|c|}
\hline Vie de saint & 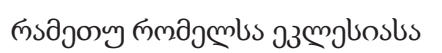 & En effet, une église qui n'a pas \\
\hline Georges, ${ }^{\mathrm{b}}$ ch. $x$ & 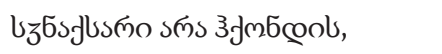 & de Synaxaire, - même si elle \\
\hline$(\$ 24$ chez & 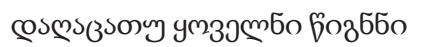 & possède tous les livres, - elle \\
\hline Peeters). & 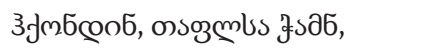 & mange du miel, mais son palais \\
\hline & 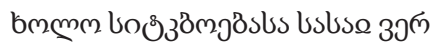 & ne ressent pas de suavité (cf. \\
\hline & 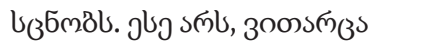 & Ps. $118: 103)$. Il est, comme \\
\hline & 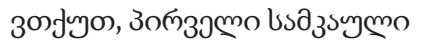 & nous avons dit, le premier \\
\hline & 0зलmgloobse. & ornement $^{\mathrm{c}}$ de l'Eglise. \\
\hline
\end{tabular}

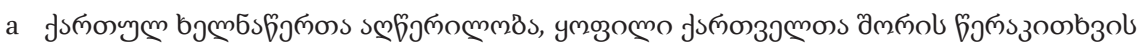

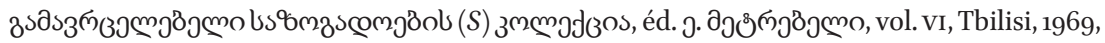

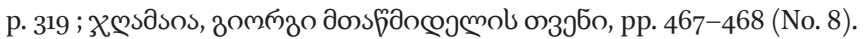

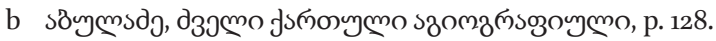

c On notera que dans sa traduction française B. Martin-Hisard privilège le contexte immédiat du passage de la Vie et remplace « ornement » par « fondement », le dernier terme étant utilisé quelques lignes avant (B. Martin-Hisard, « La Vie de Georges l'Hagiorite (1009/1010-29 juin 1065). Introduction. Traduction du texte géorgien. Notes et éclaircissements », dans : RÉ $B$, 64-65 (2006-2007), p. 52-53), même si tous les manuscrits donnent bsaz̧зymo « ornement » ; elle se rapproche ainsi de la traduction latine de Peeters, « ecclesiarum fundamentum » (Peeters, « Histoires monastiques géorgiennes », p. 95).

On peut relever trois points communs de caractère littéraire entre les pas-

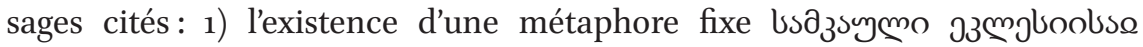
«ornement de l'Église»; 2) l'emploi de cette métaphore pour désigner figurativement un livre liturgique; 3 ) le fait que le mot 03 mglos «église» signifie l'Eglise dans le sens théologique et non pas géographique ou matériel. D'ailleurs, l'expression du colophon rentre parfaitement dans le contexte du langage poétique et figuré que saint Georges l'Hagiorite emploie lorsqu'il se réfère à son œuvre de traduction du grec en géorgien.

\section{En guise de conclusion}

Les résultats qui peuvent être tirés de notre enquête anecdotique sont d'une valeur historique plutôt négative : il n'existe pas d'église de nom de Kaouvi, riche en manuscrits grecs de valeur. En conséquence, le colophon du ms. Iviron 69 ne peut pas être considéré comme une évidence pour les liens entre la Laure 
géorgienne d'Iviron et le monastère de Kolobou à l'époque de Georges l'Hagiorite. ${ }^{13}$

13 Que de tels liens existaient entre ces deux monastères du vivant de saint Georges et en général tout au long du XI ${ }^{\mathrm{e}}$ siècle, nous l'apprenons des archives d'Iviron, publiées et commentées par Jacques Lefort, Nicolas Oikonomidès et Denise Papachryssanthou (cf. spécialement les documents $N^{\circ} 3^{2}$ et $N^{\circ} 33$ qui parlent des redevances financières de Kolobou envers Iviron : Actes d'Iviron, vol. II, Du milieu du XI e siècle à 1204, éd. J. Lefort, N. Oikonomidès, D. Papachryssanthou, V. Kravari, H. Métrévéli (Archives de l'Athos, 16), Paris, 1990, pp. 80-91). On verra un résumé exhaustif sur les relations entre Iviron et Kolobou dans : Actes d'Iviron, vol. I, Des origines au milieu du XI $I^{e}$ siècle, éd. J. Lefort, N. Oikonomidès, D. Papachryssanthou, H. Métrévéli (Archives de l'Athos, 14), Paris, 1985, pp. 28-32. 